\title{
Structural Investigation of the Capsular Polysaccharide Produced by Cryptococcus Isolated from Sea Water
}

\author{
Koichi OKUTANI*
}

(Accepted December 22, 1983)

\begin{abstract}
Cryptococcus No. 7 strain, isolated from sea water, produced a extracellular polysaccharide when grown on media containing sucrose. Purification of the polysaccharide has been accomplished by precipitation with Cetavlon and ethyl alcohol. Purified polysaccharide gave a homogeneous peak in Sepharose 4B column chromatography. Homogeneity of this substance also confirmed by ultracentrifugal analysis and electrophoresis, Chemical analyses showed the absence of aminosugar, amino acid, and protein.

Preliminary structural studies on the polysaccharide suggest that it consists of the eight saccharide repeating unit having xylose, mannose, and glucuronic acid residues, in the ratio of $3: 3: 2$, to which must be added $O$-acetyl group. Two units of 3-O-(glucopyranosyluronic acid)xylose are present as side chains, and these glucuronic acid residues occur as the terminal end groups. Two residues of $(1,2,3)$ linked mannose must be branching points in the main chain.
\end{abstract}

The present author isolated a fungal strain of the genus Cryptococcus in the sea water environment. This strain grows and produces dense capsules equally well in both media prepared with sea water and fresh water. The knowledge of marine occurring yeasts has been limited and only within recent years have there been extensive studied on this subject. Cryptococcus species are often found in the marine environments not only in estuarine but also in the open ocean and the deep sea waters and sediments. ${ }^{1-5}$ )

Strains of the genus Cryptococcus are known to produce capsules which are quite similar in composition and contain mainly xylose and mannose, with smaller amount of glucuronic acid and galactose. ${ }^{6}$ Interesting from its fungal pathogenicity to man, production and properties of an extracellular polysaccharide from Cryptococcus neoformans have been intensively investigated..$^{7-8)}$ However, no information was found about the chemical strucure of the capsular polysaccharide from marine isolates.

This report presents the results of the preliminary studies on the structure of the capsular polysaccharide produced by Cryptococcus No. 7 strain isolated from sea water.

\section{Materials and Methods}

\section{General Methods}

Optical rotations were measured at $25^{\circ} \mathrm{C}$ with a
Jasco J-20C automatic recording spectropolarimeter. Ultracentrifugal analyses were performed with Hitachi 282 analytical ultracentrifuge. Sedimentation patterns were made on $0.1 \mathrm{M}-\mathrm{NaCl}$ solution.

Electrophoresis were performed using a $1 \%$ solution on cellulose acetate strips (Sartorius $11200,27 \times 145 \mathrm{~mm})$ in veronal buffer $(\mathrm{pH} 8.6)$ at $150 \mathrm{~V}$ for $30 \mathrm{~min}$, and then developed in Alcian Blue.

${ }^{18} \mathrm{C}-\mathrm{NMR}$ spectra were obtained on a Hitachi $\mathrm{R}-900(22.6 \mathrm{MHz})$ spectrometer at $75^{\circ} \mathrm{C}$ in sample tube of $8 \mathrm{~mm}$. A solution of concentration $100 \mathrm{mg} / 1.2 \mathrm{ml}$ was made up in $\mathrm{H}_{2} \mathrm{O}$, with $\mathrm{TSP}_{4}$ (3-trimethylsilyl-propionic acid- $\mathrm{d}_{4}$ sodium salt) added as the internal reference. ${ }^{18} \mathrm{C}$-chemical shifts were measured relative to TSP $_{-} \mathrm{d}_{4}$ but are reported in parts per million relative to TMS (tetramethylsilane). The ${ }^{13} \mathrm{C}$-nuclei of TMS are $1.8 \mathrm{ppm}$ less shielded than those of TSP- $\mathrm{d}_{4}$.

Infrared (IR) spectra were obtained using the $\mathrm{KBr}$ pellet technique in a Jasco A-302 spectrophotometer.

The free acid form ( $\mathrm{H}$-form) of the polysaccharide was obtained by passing a solution of the native polysaccharide ( $\mathrm{Na}$-form) through a column of Amberlite IR-120 ( $\left.\mathrm{H}^{+}\right)$resin, followed by dialysis and freeze-drying. Deacetylation of the polysaccharide was achieved by treatment with $0.1 \mathrm{M}-\mathrm{NaOH}$ solution for $4 \mathrm{hr}$ at $30^{\circ} \mathrm{C}$, followed by dialysis and freeze-drying.

\footnotetext{
* Faculty of Agriculture, Kagawa University, Kagawa 761-07, Japan（奥谷康一：香川大学農学部).
} 
Paper chromatography was conducted on Whatman No. 1 paper by the descending method and in 18:3:1:4 of ethyl acetate-acetic acid-formic acid-water as the solvent (V/V). Chromatograms were developed by using the alkaline silver nitrate and aniline hydrogen phthalate reagents.

Gel-filtration chromatography was performed on a column $(2.2 \times 88.0 \mathrm{~cm})$ of Sepharose $4 \mathrm{~B}$ equilibrated in $0.1 \mathrm{M}-\mathrm{NaCl}$ solution. The sample $(7 \mathrm{mg})$ was applied to a column and eluted with $0.1 \mathrm{M}$ $\mathrm{NaCl}$ at a flow rate of $10.6 \mathrm{~m} / / \mathrm{h}$. Fractions $(5.3 \mathrm{~m} l)$ were collected and analyzed for sugar content by means of the phenol-sulfuric acid reagent."

Gas-liquid chromatography (GLC) was performed with a Hitachi 263-30 instrument with dual flame ionization detectors. Glass columns $(0.3 \times$ $200 \mathrm{~cm}$ ) were used with nitrogen as the carrier gas at a flow rate of $15 \mathrm{~m} / / \mathrm{min}$. The columns used were (1) $3 \%$ of SP-2340 on Uniport HP (100$120 \mathrm{mesh}$ ), and (2) $2 \%$ of OV-225 on Uniport HP (80-100 mesh), programmed from $190^{\circ} \mathrm{C}$ for $4 \mathrm{~min}$ and then at $4^{\circ} \mathrm{C} / \mathrm{min}$ to $250^{\circ} \mathrm{C}$. Identification of sugars was achieved by comparison with authentic samples.

Uronic acid content of the polysaccharide was estimated by the carbazole method. ${ }^{105}$ Protein determinations were done by the Lowry method. ${ }^{11}$ Amino acid and aminosugar analyses were performed with a Hitachi KLA-5 amino acid analyzer after hydrolysis with $2 \mathrm{~N}-\mathrm{HCl}$ at $100^{\circ} \mathrm{C}$ for $2 \mathrm{~h}$ in sealed tubes. Aminosugars were also analyzed by a modification of the Elson-Morgan procedure. ${ }^{12}$

\section{Preparation of the Polysaccharide}

A culture of Cryptococcus No. 7 was in the collection of this laboratory, which was originally isolated in the surface water of Seto Inland Sea, Japan. A one week-old culture on agar medium was seeded to a test tube containing $10 \mathrm{~m} l$ of broth having the following composition per liter of deionized water; Sucrose $(30 \mathrm{~g})$, Peptone $(5 \mathrm{~g})$, Meat extract $(3 \mathrm{~g}), \mathrm{NaCl}(1 \mathrm{~g})$. The culture was incubated for 3 days at $25^{\circ} \mathrm{C}$ and distributed to $250 \mathrm{ml}$ of agar plate $(18 \times 26 \mathrm{~cm})$ having the same medium supplemented with agar $(1.5 \%)$. These were incubated under the same conditions. The viscous materials containing fungal cells were harvested, and diluted with water containing $1 \%$ of phenol. This suspension was then centrifuged for $1 \mathrm{~h}$ at $30,000 \mathrm{rpm}$ with a Hitachi 55P-72 ultracentrifuge fitted with rotor type RP42. The clear supernatant liquior was separated, and the polysaccharide was precipitated with $5 \%$ Cetavlon solution. The precipitate was isolated by decantation and centrifugation, washed with a small volume of cold water, and dissolved in $4 \mathrm{M}-\mathrm{NaCl}$ solution. The polysaccharide was reprecipitated by pouring the solution into 2 vol. of ethanol, with stirring. The purified polysaccharide was isolated by centrifugation, dissolved in water, and then dialyzed for 2-3 days against running water. Freeze-drying of this solution yielded $280 \mathrm{mg}$ of the polysaccharide per $1 l$ of the medium.

\section{Sugar Analysis}

The polysaccharide $(20 \mathrm{mg})$ was hydrolyzed with $10 \mathrm{~m} l$ of $2 \mathrm{M}$-trifluoroacetic acid (TFA) overnight at $100^{\circ} \mathrm{C}$. The acid was removed by several evaporations with water under diminished pressure. The sugar released was identified by paper chromatography and GLC. On GLC analysis, a portion of the hydrolyzate was reduced with sodium borohydride and the mixture was made neutral with Amberlite IR-120 $\left(\mathrm{H}^{+}\right)$cation exchange resin and filtered. The filtrate was evaporated three times with methanol in order to remove borate ion. The product was acetylated with acetic anhydride-pyridine. The alditol acetates of sugar were analyzed by GLC (column 1).

Total sugar analyses were performed by methanolysis. Methanolysis of a sample $(20 \mathrm{mg})$ with $5 \%$ methanolic hydrogen chloride and neutralization with silver carbonate, and subsequent treatment with sodium borohydride in anhydrous methanol reduced the uronic acid residues in the polysaccharide. ${ }^{18-14)}$ Carboxylreduced sugars were further hydrolyzed with $2 \mathrm{M}$-TFA overnight at $100^{\circ} \mathrm{C}$. The sugar released was derivatized to alditol acetate and identified by GLC (column 1).

\section{Methylation Analysis}

The polysaccharide $(200 \mathrm{mg})$ was dissolved in dry dimethylsulfoxide $(20 \mathrm{ml})$. Methylation was performed by the Hakomori procedure. ${ }^{15)}$ After removal of the excess of the reagents by dialysis, the methylated polysaccharide was isolated by freeze-drying. Subsequent Purdie treatment ${ }^{182}$ yielded a methylated polysaccharide that showed no hydroxyl group absorption in the infrared spectrum. A portion of this methylated polysaccharide was reduced overnight with lithium alminum hydride in refluxing tetrahydrofuran. This material showed the absence of carbonyl absorption in the IR spectrum. 
Partial, Acid Hydrolysis

The polysaccharide $(100 \mathrm{mg})$ was hydrolyzed with $0.05 \mathrm{M}$-TFA $(10 \mathrm{ml})$ for $15 \mathrm{~h}$ at $100^{\circ} \mathrm{C}$. After removal of the acid by evaporation with several portions of water, the hydrolyzate was dialyzed against water $(1 l)$. After concentration, the dialyzate was fractionated by paper chromatography. Pure oligosaccharide was obtained on a Whatman 3MM paper $\left(R_{\mathrm{g} 10} 0.90\right)$.

\section{Periodate Oxidation}

Deacetylated polysaccharide $(180 \mathrm{mg})$ was subjected to periodate oxidation with $0.05 \mathrm{M}$-sodium metaperiodate $(100 \mathrm{~m} l)$ for 3 days at $5^{\circ} \mathrm{C}$ in the dark. After the excess periodate was decomposed with ethylene glycol $(20 \mathrm{ml})$, the mixture was dialyzed overnight against water. The material remaining was reduced overnight with sodium borohydride $(200 \mathrm{mg})$ and the excess of hydride was decomposed with acetic acid. The solution was dialyzed and then freeze-dried, yielding $96 \mathrm{mg}$ of the derived polyalcohol.

A part of this polyol $(15 \mathrm{mg})$ was subjected to total sugar analysis by methanolysis as described above. The other part of this polyol $(81 \mathrm{mg})$ was subjected to Smith degradation using $0.5 \mathrm{M}$-TFA overnight at room temperature and then dialyzed against water. The dialyzed materials were isolated and analyzed by paper chromatography and by GLC (column 1) as their alditol acetates. Residual polysaccharide remained in the dialysis sac was then subjected to hydrolysis with $2 \mathrm{M}$ TFA overnight at $100^{\circ} \mathrm{C}$. Sugar components were analyzed.
Results and Disèussion

After purification by precipitation with ethanol and Cetavlon, the capsular polysaccharide from Cryptococcus No. 7 strain gave a homogeneous peak in gel chromatography on Sepharose 4B. The purified polysaccharide had $[\alpha]_{589}+22.5^{\circ}$ (C 0.2 , water). Homogeneity of this material also confirmed by ultracentrifugal analysis and electrophoresis. The ultracentrifugal patterns of this polysaccharide indicated the presence of one component (Fig. 1). Approximate sedimentation coefficient was calculated by the standard method ${ }^{17)}$ to be $2.85 \mathrm{~S}$ for this polysaccharide. The polysaccharide gave a single band in electrophoresis on cellulose acetate strips.

The ${ }^{18} \mathrm{C}-\mathrm{NMR}$ spectrum of the polysaccharide confirmed the presence of the O-acetyl group $\left(\mathrm{CH}_{3}\right.$ at $21.3 \mathrm{ppm}$ ). As shown in Fig. 2, the IR spectra of the native polysaccharide showed absorption bands at $1730 \mathrm{~cm}^{-1}$ and $1250 \mathrm{~m}^{-1}$, attributable to ester and fatty acid ester, respectively. After deacetylation, the disappearance of the band at $1250 \mathrm{~cm}^{-1}$ was observed. These observations support the presence of acetyl group in this polysaccharide. The band at $1730 \mathrm{~cm}^{-1}$ was found in the spectrum of deacetylated polysaccharide of $\mathrm{H}$-form but not in that of Na-form, indicating the presence of carboxyl groups in this polysaccharide.

Chemical analyses showed the presence of uronic acid and the absence of aminosugar, amino acid, and protein.

\section{Sugar Analyses}

Hydrolysis of this polysaccharide, followed by

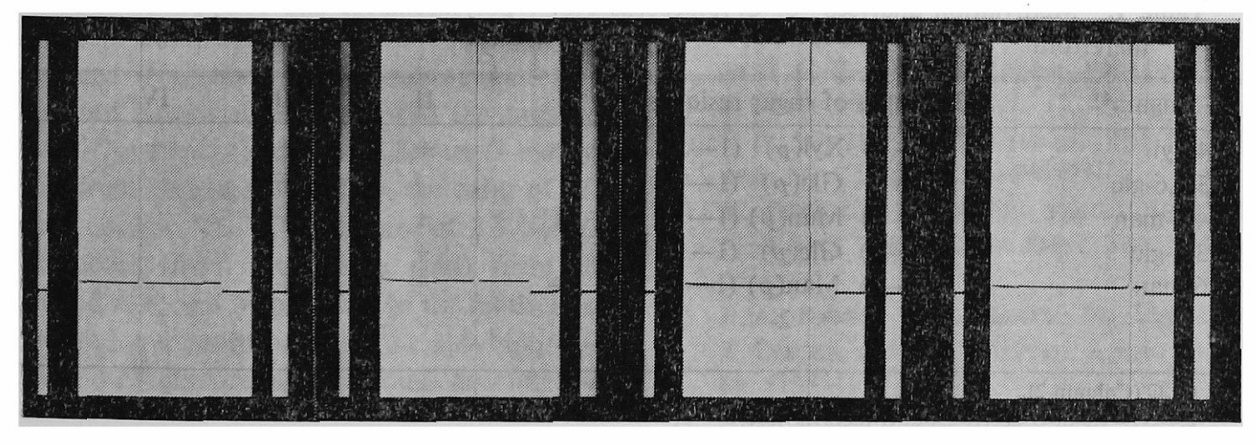

\section{$128 \mathrm{~min} \quad 72 \mathrm{~min} \quad 36 \mathrm{~min} \quad 18 \mathrm{~min}$}

Fig. 1. Ultracentrifugal patterns for purified polysaccharide.

Sedimentation patterns were obtained at various times after the rotor had reached at maximum speed of $60,000 \mathrm{rpm}$ at $25^{\circ} \mathrm{C}$ in $0.1 \mathrm{M}-\mathrm{NaCl}$ solution. Sedimentation is from right to left. 


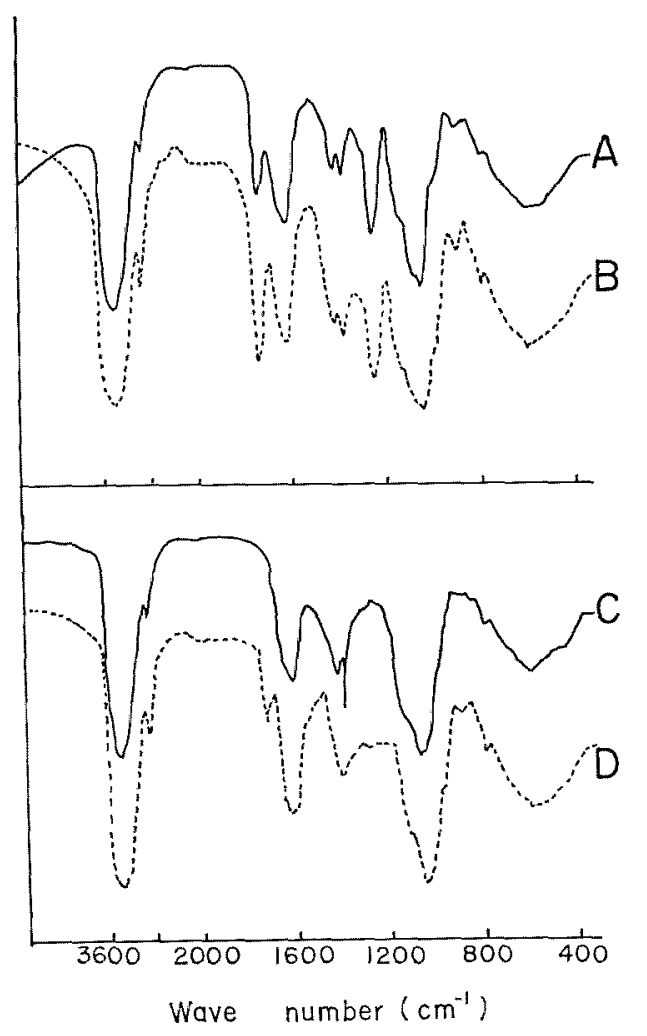

Fig. 2. IR spectra of the purified polysaccharide. Native polysaccharide A: Na-form B: $\mathrm{H}$-form

Deacetylated polysaccharide C: Na-form D: H-form paper chromatography showed the presence of xylose and mannose. The identity of these sugars were confirmed by GLC (column 1) after their conversion into alditol acetates. The presence of glucuronic acid was confirmed by total sugar analysis using the technique of methanolysis. Quantitative estimation of sugars by GLC analyses showed the presence of xylose, mannose, and glucuronic acid in the ratio of $3: 3: 2$. The estimation of uronic acid content of the polysaccharide by the method of carbazole-sulfuric acid showed the presence of $26 \%$ of uronic acid as glucuronic acid. No hexosamine and protein were detected.

\section{Methylation Analyses}

Methylated and carboxyl-reduced polysaccharide was hydrolyzed and derived sugars were converted into alditol acetates. GLC analysis gave peaks corresponding to 2,4-di- $O$-methylxylose, 2,4,6-tri$O$-methylmannose, 2,3,4-tri- $O$-methylglucose, and 4,6-di-O-methylmannose (Table 1 , column II), and found to be present in the ratio of $3: 1: 2: 2$. Methylation analysis support the concept of a eight sugar repeating unit.

When a sample of the fully methylated polysaccharide was hydrolyzed, no 2,3,4-tri- $O$-methylglucose (which would have arisen from glucuronic acid in the original carbohydrate) was found (Table 1, column I). The loss of 2,3,4-tri- $O$ methylglucose and the appearance of it after the methylated polysaccharide had been carboxylreduced, suggested that the 2,3,4-tri- $O$-methylglucose was derived from glucuronic acid. Methylated and carboxyl-reduced polysaccharide was

Table 1. Methylation analyses* of Cryptococcus No. 7 polysaccharide and compounds derived therefrom

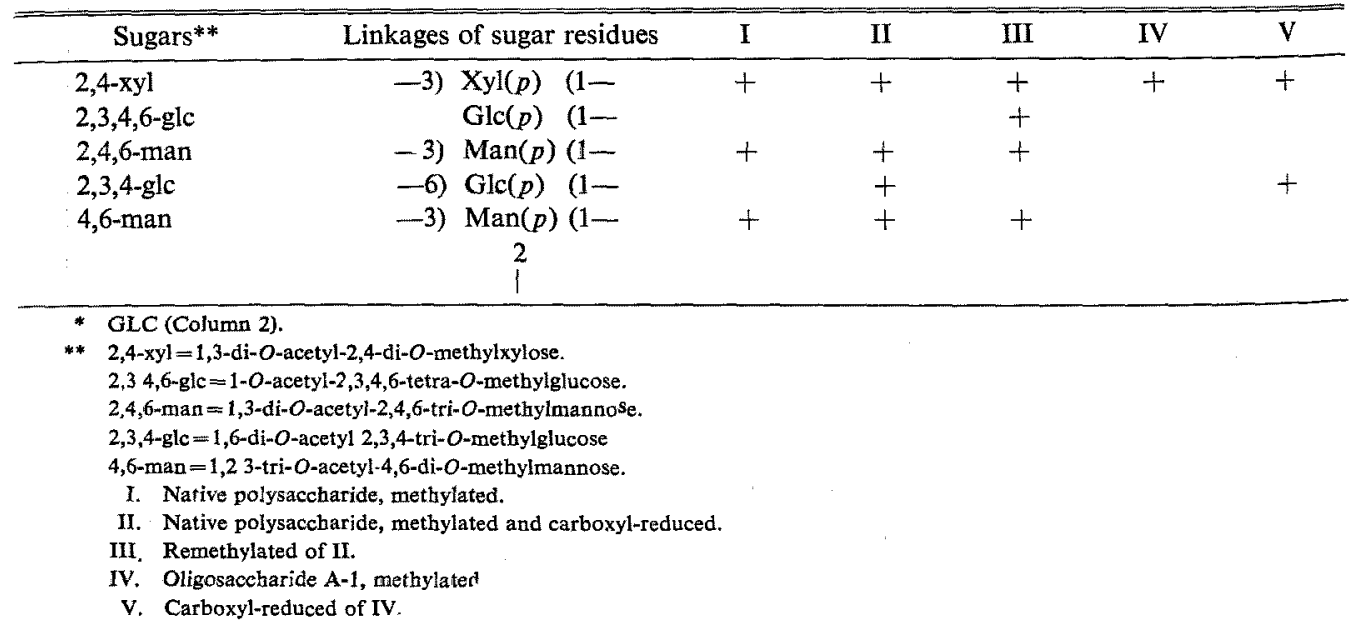


remethylated by the Hakomori procedure, and the product hydrolyzed, and the derived sugars were converted into alditol acetates. The alditol acetates of 2,4-di- $O$-methylxylose, 2,3,4,6-tetra- $O$ methylglucose, 2,4,6-tri- $O$-methylmannose, and 4,6-di-O-methylmannose were identified (Table 1 , column III). The appearance of 2,3,4,6-tetra- $O$ methylglucose and the loss of 2,3,4-tri-O-methylglucose, after the methylated and carboxyl-reduced polysaccharide had been remethylated, confirmed that the 2,3,4-tri- $O$-methylglucose was derived from glucuronic acid.

Methylation analyses show that glucuronic acid is the terminal glycosyl group of the side chain, and the presence of 4,6-di-O-methylmannose indicates that this mannose residue is a branch point in the chain. Methylation analyses show that the xylosyl mannosyl, and glucuronosyl residues are all in the pyranose form.

\section{Partial Acid Hydrolysis}

Partial hydrolysis gave an oligosaccharide (A-1) together with a mixture of monosaccharides. Component A-1 was separated and purified by paper chromatography. On hydrolysis, oligosaccharide A-1 gave xylose only. Total hydrolysis of A-1 gave glucose and xylose in the ratio of $1: 1$. The absence of glucose (from glucuronic acid) from the hydrolyzate, and its presence after total hydrolysis, show that the glucuronic acid is attached to xylose as the aldobiouronic acid.

A portion of A-1 was methylated by the Hakomori method. ${ }^{152}$ Hydrolysis and subsequent conversion into alditol acetates showed the presence of 2,4-di-O-methylxylose (Table 1, column IV). Another portion of the methylated A- 1 was reduced with lithium alminum hydride in refluxing tetrahydrofuran. Hydrolysis and subsequent conversion into alditol acetates showed the presence of 2,4-di-O-methylxylose and 2,3,4-tri-O-methylglucose (from glucuronic acid) in the ratio of 1:1 (Table 1, column V). The absence of 2,3,4-tri- $O$ methylglucose (from glucuronic acid) from the methylated A-1, and its presence in the methylated and reduced A-1 confirmed that the aldobiouronic acid was 3-O-(glucopyranosyluronic acid)-xylose.

\section{Periodate Oxidation}

Periodate oxidation of the deacetylated polysaccharide, and subsequent reduction, yielded a polyalcohol. Total sugar analysis of the derived polyol showed the presence of xylose and mannose in the ratio of 1:1, i.e., loss of glucuronic acid. This result showed that the glucuronic acid had cotiguous hydroxylgroups, thus confirming the results of the methylation analysis. As only the terminal sugar unit of the polysaccharide was susceptible to periodate oxidation, it was selectively removed by Smith degradation, to give a polysaccharide (P-1) having xylose and mannose, in the ratio of $1: 1$ as the constituent. Loss of the glucuronic acid residues involved the appearance of the xylose residues as the terminal sugar unit of the side chain.

Compound P-1 (50 mg) was subjected to a second periodate oxidation, and subsequent reduction, yielded a poly alcohol $(50 \mathrm{mg})$, analysis of which gave xylose and mannose, in the ratio of $1.8: 3$. Smith hydrolysis of the polyol with $0.5 \mathrm{M}$ TFA overnight at room temperature, followed by dialysis and freeze-drying, gave polysaccharide $(\mathrm{P}-2)$. Hydrolysis of $\mathrm{P}-2$, and subsequent derivatization into alditol acetate showed xylose and mannose, in the ratio of $1.8: 3$, i.e., there had been a loss of approximately one unit of xylose. The amount of xylose residues obtained in the hydrolysis of P-2 were higher than expected. No explanation is yet at hand, but one possibility is that the first Smith hydrolysis of the polyol using $0.5 \mathrm{M}$ TFA overnight at room temperature was unsuccessful.

The structural determinations of this purified polysaccharide are presently in progress in this laboratory.

\section{References}

1) J. V. Bhat and N. Kachwalla: Proc. Indian Acad. Sci. Sect. B. 41, 9-15 (1955).

2) J. W. Fell and N. V. UdeN: in "Symposium on Marine Microbiology" (ed. by C. H. OPPENHEIMER), C. C. Thomas, Illinois, 1963, pp. 329-340.

3) J. Fell: Bull. Mar. Sci., 17, 454-470 (1967).

4) S. P. Meyers, D. G. AhEARN, and F. J. Roth: Bull. Mar. Sci., 17, 576-596 (1967).

5) K. Tsubaki: in "Marine Microbiology" (ed. by N. TAGA), Tokyo Univ. Press, Tokyo, 1974, pp. 65-81.

6) P. A. Rebers, S. A. Baker, M. Heidelberger, Z. Dische, and E. E. Evans: J. Am. Chem. Soc., 80, 1135-1137 (1958).

7) A. K. Bhattacharjee, K. J. Kwon-Chung, and C.P.J. Glaudemans: Carbohydr. Res., 73, 183192 (1979).

8) R. Cherniak, E. Reiss, M. E. Slodki, R. D. Plattner, and S. O. Blumer: Mol. Immunol, 17, 1025-1032 (1980).

9) M. Dubors, K. A. Gilles, J. K. Hamilton, P. A. Rebers, and F. SMTth: Anal. Chem., 28, 350- 
356 (1956).

10) Z. Dische: J. Biol. Chem., 167, 180-198 (1947).

11) O. H. Lowry, N. J. Rosebrough, A. L. FARr, and R. J. RANDALI: J. Biol. Chem., 193, 265175 (1951).

12) C. J. M. Rondle and W. T. J. MorGaN: Biochem. $J$, 61, 586-589 (1955).

13) G. G. S. Dutton, K. L. Mackie, A. V. Savage, and M. D. Stephenson: Carbohydr. Res., 66, 125-131 (1978).
14) G. G. S. Dutton and M. T. YANG: Carbohydr. Res., 59, 179-192 (1977).

15) S. HaKomori: J. Biochem., 55, 205-208 (1964).

16) T. Purdie and J.C. Irvine: J. Chem. Soc., 85, 1049-1070 (1904).

17) G. Matsumura: in "Method for Biochemical Analysis" (ed. by Japanese Biochemical Society), Vol. 4, Tokyo Kagaku Dozin, Tokyo, 1976, pp. $448-460$. 